EVALUACIÓN COMPRENSIVA DE UN ÁREA DE FORMACIÓN DOCENTE: EL EJE DE CONTEXTOS PROFESIONALES

\title{
Evaluación comprensiva de un área de formación docente: el eje de contextos profesionales ${ }^{1}$
}

\author{
Comprehensive evaluation of a teacher education \\ area: the axis of professional contexts
}

Avaliação abrangente de uma área de formação de
professores: o eixo de contextos profissionais

Recibido: mayo de 2013

Aprobado: agosto de 2013

María Isabel Ramírez Garzón ${ }^{2}$

\section{Resumen}

Este estudio de caso busca comprender el eje de contextos profesionales a partir de la pregunta: desde las percepciones de los actores del programa, ¿cuál es la pertinencia y los efectos que tiene la formación que ofrece el eje en el futuro desempeño de los profesores de matemáticas? El abordaje de esta pregunta se realizó utilizando como técnicas de recolección de información encuestas y entrevistas: estudiantes, docentes, administrativos, egresados y empleadores, constituyeron las principales fuentes de información. Los resultados ofrecen consideraciones que permiten conocer y comprender el valor de esta experiencia educativa en la formación de profesores de matemáticas en el contexto colombiano.

Palabras clave: Enseñanza; análisis y reflexión sobre la enseñanza; evaluación de programas educativos; evaluación comprensiva; profesor; formación de profesores de matemáticas; eje de contextos profesionales.

\begin{abstract}
This case study seeks to understand the core of professional contexts from the question: from the perceptions of the actors in the program, what is the relevance and the effects that the training offered by the shaft in the future performance of teachers math? The approach to this question was performed using as data collection techniques surveys and interviews: students, teachers, administrators, alumni and employers, were the main sources of information. The results provide considerations to know and understand the value of this educational experience in the training of teachers of mathematics in the Colombian context.
\end{abstract}

Keyword: education, analysis and reflection on teaching, evaluation of educational programs, comprehensive evaluation, teacher, math teacher training, professional contexts axis.

1 Artículo de Investigación.

2 Universidad de los Andes. Doctorado en Educación. Bogotá, Colombia. Contacto: mi.ramirez78@uniandes.edu.co 


\section{Resumo}

Este estudo de caso visa compreender o núcleo de contextos profissionais da questão: a partir das percepções dos atores no programa, qual é a relevância e os efeitos que a formação oferecida pelo eixo no futuro desempenho dos professores matemática? A abordagem a esta pergunta foi realizada utilizando como técnicas de coleta de dados de pesquisas e entrevistas: estudantes, professores, administradores, ex-alunos e empregadores, eram as principais fontes de informação. Os resultados fornecem considerações a conhecer e compreender o valor desta experiência de ensino na formação de professores de matemática no contexto colombiano.

Palabras clave: educação, análise e reflexão sobre o ensino, a avaliação de programas educacionais, avaliação abrangente, professores, formação de professores de matemática, eixo contextos profissionais.

\section{Introducción}

Son limitadas las propuestas de evaluación comprensiva para el eje de contextos profesionales de la Licenciatura en Educación Básica con énfasis en Matemáticas (LEBEM) de la Universidad Distrital (UD) que brinden información desde las percepciones de todos los grupos de interesados frente a dos aspectos: la pertinencia y los efectos que la formación de este eje tiene en el futuro desempeño profesional de los profesores de matemáticas egresados de este programa.

El presente estudio parte de reconocer que el programa de LEBEM de la UD ha cumplido a cabalidad con los procesos de acreditación de calidad exigidos por el marco legal colombiano; como resultado el MEN, el CNA, la misma IES a la que pertenece el programa y la LEBEM la han reconocido y declarado en la última década como un programa de formación de profesores de matemáticas de alta calidad en Colombia. En ese sentido, el estudio no tiene la pretensión de cuestionar la calidad del programa; por el contrario, busca a partir de la realización de una evaluación comprensiva de uno de sus componentes (eje de contextos profesionales), aportar a la autoevaluación del mismo en la perspectiva de contribuir con mayores elementos de análisis.
Además de un posible aporte a la autoevaluación del programa, este estudio tiene interés en aportar al campo de la investigación en educación matemática en Colombia. La búsqueda de información en torno a este tema arrojó como resultado encontrar escasa investigación existente respecto a la formación de profesores de matemáticas.

Una de las problemáticas que en el contexto colombiano se plantean a la investigación en educación matemática y a la formación de profesores [...] es que existe un débil flujo, conocimiento y utilización de la información más actualizada sobre los avances de la comunidad de educadores matemáticos, sus desarrollos teóricos y empíricos. (Lurduy, 2009, p. 166).

Así mismo no encontré información relacionada con la evaluación de programas de formación de profesores de matemáticas de última generación desde la perspectiva teórica asumida en este estudio. Uno de los autores que ha indagado acerca de este tema afirma:

La investigación sobre formación de profesores de matemáticas es muy reciente en nuestro medio, pero a pesar de los esfuerzos grupales y personales de investigadores por desarrollar y gestionar dichos programas de formación, es evidente que sobre los

2 Esta expresión refiere a los programas desarrollados desde el año 2000 hasta el día de hoy. 
programas de formación de profesores de matemáticas de la última generación, sobre la práctica docente o sobre las competencias desarrolladas en esa formación, la investigación, la producción académica, los artículos en revistas reconocidas, entre otros, es inexistente. (Lurduy, 2009, p. 166).

Finalmente, encontré que las evaluaciones frente a aspectos puntuales de la formación de profesores de matemáticas tales como el papel que juega lo social y lo humano en su formación son escasas. $\mathrm{Al}$ respecto Wood (2008) citado en Lurduy (2009) relaciona los aspectos puntuales de la formación de profesores de matemáticas como temáticas que ameritan mayor investigación, entre ellas la evaluación de programas en este campo.

Atendiendo a las razones mencionadas anteriormente, se consideró pertinente y valioso el desarrollo del presente estudio cuyo propósito fue el desarrollo de una evaluación comprensiva del eje de contextos profesionales de la LEBEM de la UD a partir de las percepciones de los actores del programa respondiendo a la pregunta: ¿cuál es la pertinencia y los efectos que tiene la formación que ofrece el eje en el futuro desempeño de los profesores de matemáticas? En ese sentido, se considera este trabajo como una contribución a un campo poco explorado en la última década tanto en el contexto nacional como en el internacional.

\section{Marco de referencia conceptual}

Los aspectos tomados en cuenta conciernen a la formación de profesores de matemáticas en la LEBEM y la evaluación de programas en educación.

La formación de docentes de matemáticas en la LEBEM. Lurduy (2009) presenta la LEBEM como un programa de formación de profesores de matemáticas que cuya propuesta curricular ha adoptado una perspectiva de la pedagogía como disciplina reconstructiva, crítica y compleja, además de una perspectiva sistémica de la didáctica de las matemáticas y de la educación matemática como disciplina científica. Es decir, se concibe como un proyecto de investigación desde una mirada interdisciplinar soportado en investigaciones que refieren las concepciones y creencias del profesor y del profesor de matemáticas, las innovaciones curriculares y el currículo como proyecto de investigación, la investigación y conceptualización en formación de profesores de matemáticas, la construcción del conocimiento profesional del profesor de matemáticas, el profesor reflexivo y el profesor investigador, y la resolución de problemas como metodología de enseñanza. El plan de estudios se estructura en cuatro ejes de formación: matemáticas escolares, pensamiento matemático avanzado, práctica en el aula de clase y contextos profesionales, este último, objeto de estudio en la presente investigación.

Con esta descripción de la licenciatura y teniendo presente que es un programa que ha sido reconocido y acreditado de alta calidad por el CNA en dos ocasiones $(2005,2010)$ en sus casi doce años de trayectoria, además de que ha sido pensado para atender a las necesidades educativas del contexto colombiano y es reconocido como una innovación dentro de la educación superior en Colombia, llevé a cabo la evaluación de uno de sus componentes, el eje de contextos profesionales, como espacio de formación en el que "la función de la pedagogía y de las humanidades cobra un lugar importante para la formación y desempeño del educador matemático" (Gil, 2011, p. 2).

Evaluación de programas en educación. Entiendo la evaluación de programas educativos como un proceso sistemático de recogida de información que conduce e implica la formulación de un juicio de valor acerca de un programa en la perspectiva de tomar posibles decisiones orientadas al mejoramiento de las prácticas (Jiménez, 1999).

La formulación del juicio de valor otorgado a un programa depende del enfoque evaluativo bajo el cual este se mire. Ramírez (2012) muestra en su revisión la existencia de enfoques evaluativos orientados a valorar la efectividad del programa desde el logro de objetivos así como también orientados a conocer la utilidad y los efectos de un programa de cara a las necesidades de los consumidores. Otros usan la evaluación como una herramienta que guía la toma de decisiones, otros pretenden determinar la calidad de un programa desde un conjunto de estándares establecidos o entender el programa y la calidad del 
mismo a partir de distintas interpretaciones que los actores que lo conforman pueden hacer de él. Este último enfoque corresponde a la postura teórica asumida en este estudio y se concibe como una guía metodológica que busca examinar completamente un programa a partir de recopilar y reflejar las múltiples percepciones de los grupos de actores del mismo sobre el valor de su formato, las operaciones, y sus logros. Para Stake (2001) las percepciones son entendidas como el valor que otorga cada uno de los participantes del programa al mismo de acuerdo con su interpretación de la realidad, tal interpretación está basada en su propia experiencia en él. Así, el juicio evaluativo radica entonces en un consenso de perspectivas que debe ser descubierto por el evaluador al escuchar las percepciones que representan los diferentes grupos de actores del programa. Vale la pena resaltar que esta es la razón por la cual no concentré el estudio en analizar únicamente los productos que emergen del programa, sino el conjunto completo de perspectivas.

\section{Metodología}

Esta investigación se enmarcó en el paradigma constructivista cuyo propósito fue conocer e interpretar en profundidad el eje contextos profesionales. En este sentido realicé un estudio cualitativo, del tipo estudio de caso. Un total de 293 estudiantes (75\%), el coordinador del programa, diez profesores, 37 egresados y 12 empleadores constituyeron los grupos de interesados. Ellos dieron sus percepciones acerca del eje de contextos profesionales a través de entrevistas semi-estructuradas (coordinador y profesores), encuestas (estudiantes y empleadores) y grupos focales (estudiantes); allí, narraron su experiencia dentro de la formación que han impartido, imparten, reciben o recibieron en la licenciatura y cómo esa experiencia ha incidido en su práctica o en su trayectoria profesional.

\section{Análisis de datos}

Los datos fueron analizados usando técnicas cualitativas propuestas por Stake (2010). Para responder a la pregunta de investigación del estudio, transcribí y sistematicé cada uno de los datos cualitativos obtenidos en las entrevistas semi-estructuradas y las encuestas, en la búsqueda de los temas asociados a cada una de las subpreguntas de investigación establecidas para atender a los dos aspectos en los que se enfocó esta evaluación comprensiva del eje de contextos: la pertinencia y los efectos que perciben los diferentes actores de la LEBEM.

\section{Conclusiones}

Desde los actores la ejecución del programa evidencia un diseño sólido en [1] una perspectiva teórica clara sobre el desarrollo del pensamiento complejo, [2] el valor agregado que ofrece a la formación de los licenciados: el componente de conocimientos transversales a las matemáticas que permite desarrollar trabajos de tipo interdisciplinar con la educación matemática y [3] el tipo de reflexión que propicia en el estudiante para profesor: la complejidad del ser humano y la realidad de la profesión "profesor de matemáticas". Los actores del programa consideran que el eje aporta desde tres grandes campos a la formación del profesor de matemáticas:

Formación pedagógica. Al transformar la enseñanza tradicional y enciclopédica de la pedagogía, este eje vincula todo el componente humanístico a la formación del licenciado.

Desarrollo de cinco habilidades. (a) Pensamiento crítico, da elementos para que los estudiantes puedan comprender la educación en relación con la condición del mundo. (b) Habilidades comunicativas, se espera que en el mundo profesional los profesores de matemáticas sean lectores y productores de escritos, el eje mantiene un alto nivel de exigencia desde todas las actividades que promueve respecto a la oralidad, la lectura y la escritura. (c) Trabajo en equipo, como una de las competencias que el profesional de hoy debe manejar, siendo un profesional de la docencia que todo el tiempo está interactuando con otros. (d) Capacidad para solucionar problemas; el eje se constituye en una base en la que se fundamentan elementos de análisis que permiten a los estudiantes pensar y 
resolver problemas. (e) Habilidades de investigación sobre la práctica.

Proyección del profesor. El eje le apuesta a que sus estudiantes hagan de la docencia su opción de vida. Trabaja por la construcción y la constitución de la identidad del profesor invitando al estudiante a que se piense como profesor de matemáticas y tome conciencia de las implicaciones que esto tiene para su vida profesional y también de la responsabilidad ético-moral, social y política que tiene con las vidas que va a impactar con su labor.

\section{Referencias}

Gil, D. (2011). El eje de contextos profesionales, una formación pedagógica y humana para la formación de docentes de matemáticas no segregadores. Congreso internacional de educación universitaria y prácticas educativas innovadoras. 21, 22 y 23 de septiembre de 2011. México: Universidad Nacional Autónoma de México.

Jiménez, B. (1999). Evaluación de programas centros y profesores. La evaluación de programas para la innovación. En Jiménez (Eds.). Evaluación de programas, centros y profesores (pp. 237 264). Madrid: Síntesis

Lurduy, J. O. (2009). Investigación en la formación de profesores de matemáticas. Agendas y perspectivas. En Revista Científica No.11. p. 165 178. Bogotá, Colombia: Universidad Distrital Francisco José de Caldas.

Ramírez, M.I. (2012). Evaluación comprensiva de un área de formación docente. El caso de la Licenciatura en Educación Básica con énfasis en Matemáticas de la Universidad Distrital Francisco José de Caldas. Tesis de grado obtenido no publicada. Universidad de los Andes, Bogotá, Colombia.

Stake, R. (2006). Evaluación comprensiva. En Graò (Ed.), Evaluación comprensiva y evaluación basada en estándares (pp. 141 - 165). Barcelona, España: Graó

Stake, R. (2010). Qualitative Research. Studying how things work. The Guilford Press. New York, USA 